\title{
Probiotics and MicroRNA: Their Roles in the Host-Microbe Interactions
}

\section{OPEN ACCESS}

Edited by:

Andrea Masotti,

Bambino Gesù Children Hospital

(IRCCS), Italy

Reviewed by:

Shirong Liu,

Brigham and Women's Hospital and Harvard Medical School,

United States

Meysam Sarshar

Sapienza University of Rome, Italy

*Correspondence:

Xueqin N

xueqinni@foxmail.com

xueqinni@yahoo.com

Specialty section:

This article was submitted to

Systems Microbiology,

a section of the journal

Frontiers in Microbiology

Received: 09 September 2020 Accepted: 07 December 2020

Published: 14 January 2021

Citation:

Zhao $Y$, Zeng $Y$, Zeng $D$, Wang $H$, Zhou M, Sun N, Xin J, Khalique A,

Rajput DS, Pan K, Shu G, Jing B and NiX (2021) Probiotics and MicroRNA:

Their Roles in the Host-Microbe

Interactions.

Front. Microbiol. 11:604462. doi: 10.3389/fmicb.2020.604462

\begin{abstract}
Ying Zhao', Yan Zeng', Dong Zeng ${ }^{1}$, Hesong Wang', Mengjia Zhou ${ }^{3}$, Ning Sun', Jinge Xin ${ }^{1}$, Abdul Khalique ${ }^{1}$, Danish Sharafat Rajput ${ }^{1}, K^{\prime}$ angcheng Pan', Gang Shu', Bo Jing ${ }^{1}$ and Xueqin $\mathrm{Ni}^{1 *}$
\end{abstract}

${ }^{1}$ Animal Microecology Institute, College of Veterinary, Sichuan Agricultural University, Chengdu, China, ${ }^{2}$ Guangdong Provincial Key Laboratory of Gastroenterology, Department of Gastroenterology, Institute of Gastroenterology of Guangdong Province, Nanfang Hospital, Southern Medical University, Guangzhou, China, ${ }^{3}$ Sichuan Academy of Animal Sciences, Animal Breeding and Genetics Key Laboratory of Sichuan Province, Chengdu, China

Probiotics are widely accepted to be beneficial for the maintenance of the gut homeostasis - the dynamic and healthy interactions between host and gut microorganisms. In addition, emerging as a key molecule of inter-domain communication, microRNAs (miRNAs) can also mediate the host-microbe interactions. However, a comprehensive description and summary of the association between miRNAs and probiotics have not been reported yet. In this review, we have discussed the roles of probiotics and miRNAs in host-microbe interactions and proposed the association of probiotics with altered miRNAs in various intestinal diseases and potential molecular mechanisms underlying the action of probiotics. Furthermore, we provided a perspective of probiotics-miRNA-host/gut microbiota axis applied in search of disease management highly associated with the gut microbiome, which will potentially prove to be beneficial for future studies.

Keywords: probiotics, microRNA, gut microbiota, homeostasis, host-microbe interactions

\section{INTRODUCTION}

Trillions of microbes exist in the human gastrointestinal tract, and the number of these microbes greatly exceeds the number of human body cells, which influences the physiological activities of the host throughout the lifespan (Li D. et al., 2016; Chang and Kao, 2019). This complicated ecosystem containing bacteria, fungi, viruses, and protists is called the gut microbiota. Intense exploration for understanding the relationships between host and gut microbiota has never ceased. In particular, the "dysbiosis" of gut microbiota may lead to the occurrence of various diseases of the host, ultimately damaging its health (Li D. et al., 2016; Chang and Kao, 2019). Probiotics, in sufficient doses, can restore the intestinal microecological balance and promote the healthy growth of the host (Hill et al., 2014). The consumption of probiotics is prevalent because they have the potential to become an important intervention strategy to prevent and treat various diseases (Balakrishnan and Floch, 2012; Suez et al., 2019; Yousefi et al., 2019). Although probiotics have great prospects, concerns about security and doubts about their effectiveness are always present. It is important to explain the exact molecular mechanism describing probiotics' influence on host-microbe interactions, which remains largely unknown so far. MicroRNAs (miRNAs) are 
considered to be important regulators in the host gene expression process, and some are found to be significantly related to the microbial community or specific bacteria (Hasan and Yang, 2019). Recent studies have proposed the functional role of miRNAs played in shaping gut microbiota (Moloney et al., 2019; Behrouzi et al., 2020). These host-derived miRNAs were able to enter bacterial cells to cultivate or inhibit specific bacteria (Liu et al., 2016; Spinler et al., 2019). These effects on hostmicrobe interactions suggested that miRNAs may be important participants in the molecular mechanism of probiotics action.

In this review, we first summarized the interactions between host and gut microbiota and presented a brief overview of probiotic functions and their impact on host-microbe interactions. Then, we highlighted the importance of miRNAs to host gastrointestinal function and described the correlation between miRNA expression levels and gut microbiota profiles. Next, we discussed the current understanding of the host's use of fecal miRNA and plant-derived miRNA in maintaining gut homeostasis. Finally, we proposed the potential molecular mechanism underlying how probiotics maintain gut homeostasis and proposed the concept of probiotics-miRNA-host/gut microbiota axis that can serve as a new direction for future exploration.

\section{INTERACTIONS BETWEEN HOST AND GUT MICROBIOTA}

Genes encoded by the gut microbiota are 150 times larger than the human genome, which includes an extremely rich enzyme repository (El Kaoutari et al., 2013; Rooks and Garrett, 2016; Rowland et al., 2018; Zimmermann et al., 2019). Most members of gut microbiota exert harmless or beneficial effects on the host. They provide uncoded human enzymes, participate in human metabolism, and also maintain immune homeostasis through their interactions with host cells (Rooks and Garrett, 2016; Rowland et al., 2018). For example, the bacterial fermentation products of carbohydrates are short-chain fatty acids (SCFAs) that are important energy sources for bacteria itself and host cells (Rowland et al., 2018). SCFAs also have varying regulatory functions on the host, including the enhancement of epithelial barrier function and maintenance of mucosal immunity (Rooks and Garrett, 2016; Rowland et al., 2018). Additionally, bile acids are classical examples of the interactions between host and gut microbiota. The gut microbes modified the bile acids synthesized in the liver into secondary bile acids that modulate multiple host metabolic processes and immune homeostasis (Long et al., 2017; Parasar et al., 2019). In contrast, bile acids are capable of influencing the growth of bacteria, resulting in changes in the structure of the microbial community. The gut microbiota is essential for host metabolism and immune homeostasis, and their components contribute considerably to shaping the host immune system, such as lipopolysaccharide, flagellin, peptidoglycan, etc. (Rooks and Garrett, 2016).

Interactions between the host and gut microbiota promote the establishment of a symbiotic relationship during host development. The optimal operation of a symbiotic relationship is vital to host health. But, the performance of gut microbiota is affected by various factors, including diet, lifestyle, illness, environment, hygiene, genetics, and antibiotic exposure (Li D. et al., 2016; Cristofori et al., 2018; Celiker and Kalkan, 2020). Although the composition of specific flora may change due to the stimulation of internal and external factors, the structure of the intestinal microbial community remains dynamic and stable (Coyte et al., 2015). Differences in the types of microorganisms are carried by different individuals, but the composition and function of the "core microbiome" are similar and remain relatively stable over time (Lozupone et al., 2012; Coyte et al., 2015; Milani et al., 2017; Gentile and Weir, 2018). This microbial stability is considered to play a critical role in the health of the host. It is worth mentioning that it is regarded as a sign of host intestinal health. Disruption of microbial stability often leads to "dysbiosis," whichinduces a range of diseases (Lozupone et al., 2012; Coyte et al., 2015; Li D. et al., 2016; Milani et al., 2017). Moreover, gut microbiota have the ability to trigger responses from a distance by producing metabolites (Agus et al., 2018; Allaire et al., 2018; Gentile and Weir, 2018). The crosstalk between host and gut microbes can be linked to the health status of other body organs (Gresse et al., 2017; Agus et al., 2018; Allaire et al., 2018; Gentile and Weir, 2018; Adak and Khan, 2019; Maslowski, 2019; Zhang et al., 2019). Another aspect of the crosstalk includes the detailed mechanism of host manipulation of the bacterial community that has also received extensive attention (Figure 1). The traditional pathways are mainly dominated by bile acids, antibacterial peptides, and IgA (Brown et al., 2013; Chu and Mazmanian, 2013; Liu, 2016; Parker et al., 2018). Additionally, compelling evidences supported that genetic and environmental factors contributed greatly to the occurrence of dysbiosis, which in turn promoted many diseases, such as obesity and inflammatory bowel disease (IBD; Zhou M. et al., 2017; Cuevas-Sierra et al., 2019; Celiker and Kalkan, 2020). Recent studies have proposed a novel mechanism that the host specifically controls the gut microbiota by promoting the liberation of miRNAs (Liu et al., 2016). Diet and probiotics are also valuable tools available to alter the gut microbiota (Forgie et al., 2019; Tang, 2019).

\section{PROBIOTICS AND THEIR IMPLICATIONS FOR HOST-MICROBE INTERACTIONS}

Probiotics are commercial food supplements that are widely used all over the world. It has been generally accepted that they are able to restore the disruption of gut microorganisms. In 2002, probiotics were defined by WHO as live microorganisms that confer a health benefit when administered in adequate amounts (Hill et al., 2014). At present, as shown in Figure 2, various methods, including metagenomics, transcriptomics, and metabolomics, have already been applied to describe the mechanisms of the effects of probiotics on the host-microbe interactions (Bermudez-Brito et al., 2019; Kiousi et al., 2019; Yadav and Pratyoosh, 2019; Quigley and Gajula, 2020; Sehrawat et al., 2020). The most commonly beneficial effect of the probiotic administration route is to stabilize the bacterial 


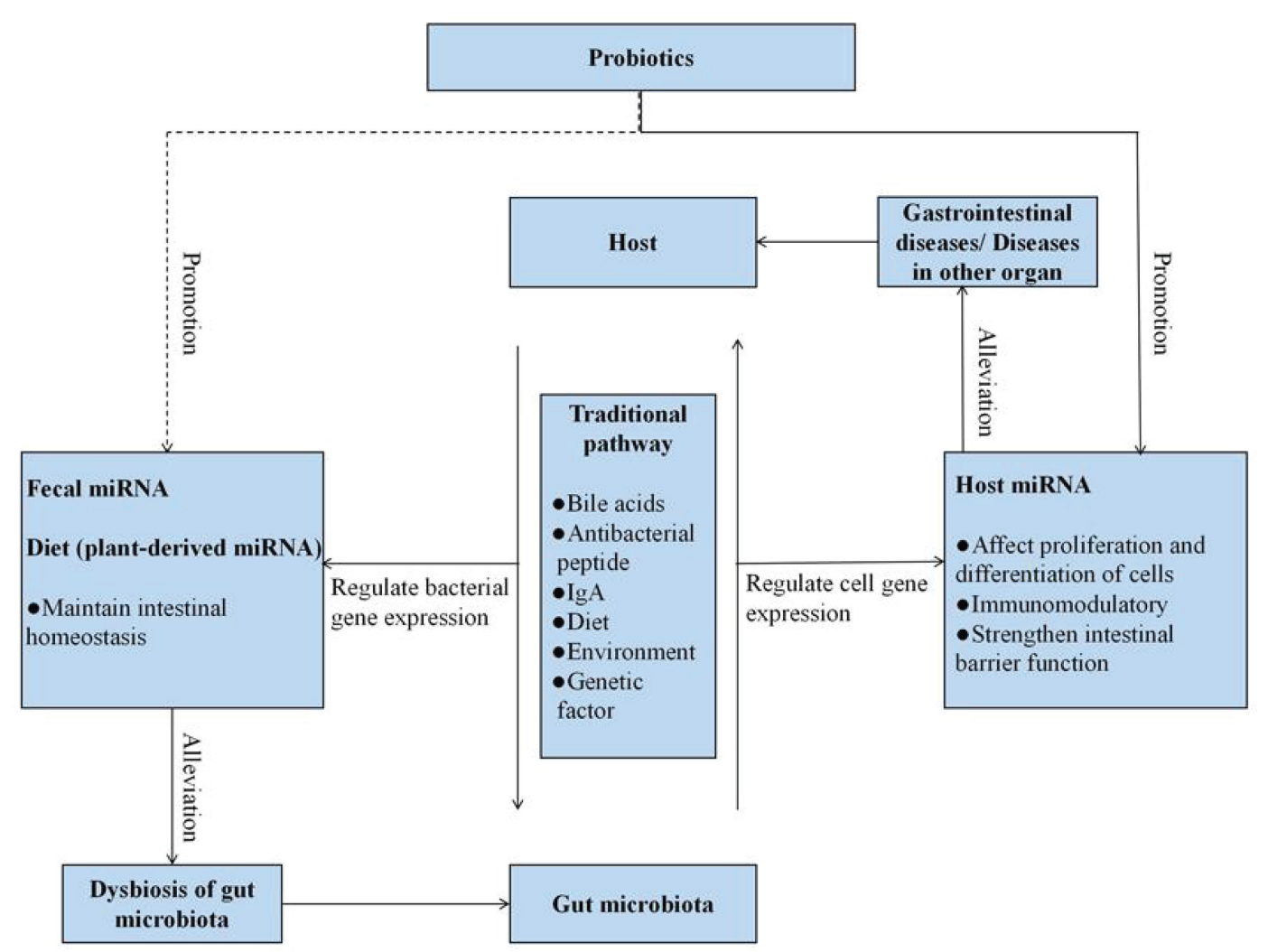

FIGURE 1 | Probiotics and miRNA, as well as their implications for host-microbe interactions. The traditional pathways involving in host-microbe interactions are mainly dominated by bile acids, antibacterial peptide, and IgA. In addition, the traditional pathways also include dietary, environmental, and genetic factors. Moreover, the host regulates bacterial gene expression via fecal miRNA and plant-derived miRNA to shape gut microbiota. Gut microbiota regulate cell gene expression via host miRNA to alleviate various gastrointestinal diseases. Probiotics have impacts on these host-microbe interactions. The dotted line represents the lack of conclusive evidence in the relationship at present.

community and restore the "signature" of gut microbiota. It mainly stems from their ability to produce bacteriocins, lower $\mathrm{pH}$, and compete with pathogens for space and nutrients (Balakrishnan and Floch, 2012; Cremon et al., 2018; Suez et al., 2019). Competitive exclusion by probiotics is based on the competition for available nutrients and adhesion sites. It has been demonstrated that purified surface adhesion proteins of probiotics can exclude pathogens. Surface adhesins of pathogens and probiotic strains are implicated in mediating the binding of microbes to the host (Du et al., 2010). Probiotics can also change the environment to gain a more favorable competitive advantage. Studies on lactobacilli and bifidobacteria have shown that these two probiotic strains could produce organic acids to lower intestinal $\mathrm{pH}$. Both organic acids and bacteriocins produced by bacteria enable the inhibition of pathogen growth (Dobson et al., 2012). On the other hand, probiotic bacteria (e.g., Lactobacillus and Bifidobacterium) have been revealed to improve barrier function. The ability of probiotics to upregulate expression levels of mucus-secretion genes may be one of the mechanisms of improving barrier function and excluding pathogens (Khoruts, 2018; Bermudez-Brito et al., 2019; Sanders et al., 2019; Yan and Polk, 2020). Furthermore, probiotic strains have been reported to directly exert immune and anti-inflammatory effects on the host. Probiotics may affect the immune system by changing the levels of metabolites, components, and DNA. They have been shown to activate immune cells, increase the production of immunoglobulins, and regulate cytokines (Khoruts, 2018; Sanders et al., 2019; Liwinski and Elinav, 2020; Yan and Polk, 2020). Several studies have demonstrated that these probioticderived factors can suppress intestinal inflammation via targeting toll-like receptor (TLR) signaling (Gómez-Llorente et al., 2010). Additionally, probiotics could also alter energy metabolism and impact intestinal physiology by releasing metabolites including the SCFAs, oligosaccharides, vitamins, amino acids, and secondary bile acids (Gura, 2014; Lee et al., 2018; BermudezBrito et al., 2019; Kiousi et al., 2019; Quigley and Gajula, 2020; Sehrawat et al., 2020). For instance, a recent study found that the SCFAs produced by Lactobacillus casei and Bifidobacterium breve could potentially be an essential regulatory effector of intestinal epithelial cell (IEC) proliferation (Matsuki et al., 2013). Currently, nutritional programs have received increasing attention; probiotic administration manipulates the gut microbiota to prevent or attenuate metabolic-related diseases (e.g., hypertension and hyperglycemia) (LeBlanc et al., 2017). 


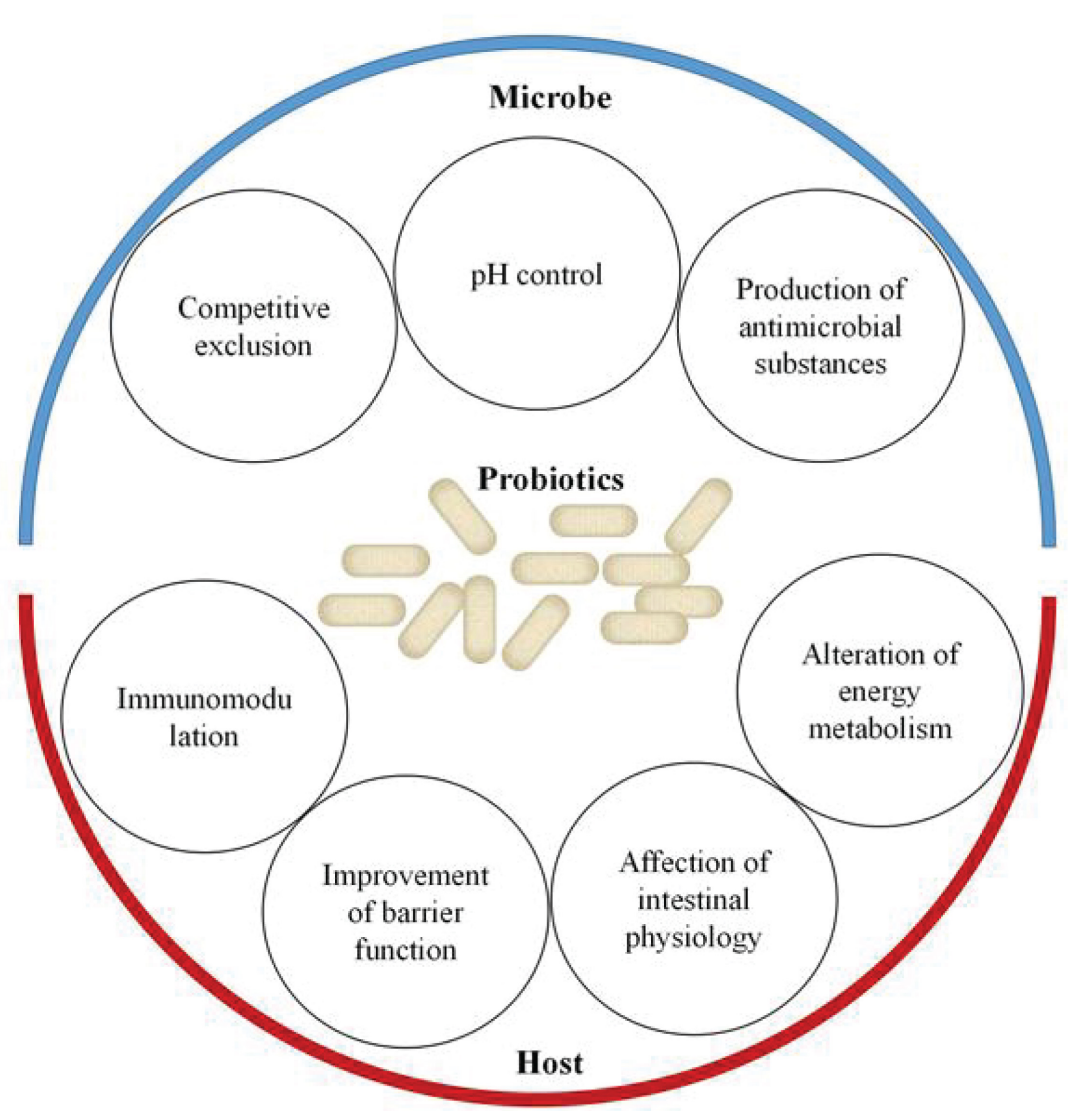

FIGURE 2 | Effects of probiotics on the host-microbe interactions. Probiotics support the stability of the bacterial community and restore the "signature" of gut microbiota through competitive exclusion, $\mathrm{pH}$ control, and production of antimicrobial substances. On the other hand, probiotics promote health benefits for the host through improvement of barrier function, immunomodulation, affection of intestinal physiology, and alteration of energy metabolism.

As probiotics can prevent and/or treat various diseases, their consumption is becoming more and more common all over the world. The growing industry shows that probiotics have a huge market prospect and the global market is expected to reach $\$ 65$ billion by 2024 (Abid and Koh, 2019). Of note, both current research and application have found some limitations of probiotics. The action level of probiotics depends on different conditions, including the strains, individuals, types of administration, or location within the intestines (Markowiak and Śliżewska, 2017; Kothari et al., 2019; Langella and Chatel, 2019; Salvucci, 2019; Wan et al., 2019). Although compelling evidence has been obtained in clinical trials, the exact molecular mechanism of probiotics that confer beneficial effects on the host remains unclear. Of late, new possibilities have been observed in different studies exploring the interaction between miRNA and microbiota and the responses of miRNA to probiotic intervention.

\section{WHAT IS MIRNA?}

MicroRNAs are endogenous, small, non-coding RNAs that are 19-25 nucleotides in length ( $\mathrm{Yu}$ et al., 2016). They are first synthesized in the nucleus, transported into the cytoplasm, and processed into mature miRNAs (Yu et al., 2016; Bartel, 2018). Mature miRNAs are loaded onto the miRNA-induced silencing complex (miRISC) and bound to the $3^{\prime}$ untranslated region (3' UTR) of mRNA to mediate post-transcriptional gene silencing (Yu et al., 2016; Bartel, 2018).

Despite complexity was added in terms of gene regulation, interest has surged in the function of miRNA molecules to mediate gene silencing to repress protein synthesis. Since the first miRNA, lin-4, was discovered in the 90s in Caenorhabditis elegans, hundreds of different miRNAs were found to play critical roles in healthy and pathological cell processes, including cellular differentiation, proliferation, signal transduction, and apoptosis (Fischer, 2015; Mohr and Mott, 2015; Yu et al., 2016; Rupaimoole and Slack, 2017; Bartel, 2018). Insights into the ability of miRNAs had made them into promising therapeutic tools (miRNA mimics) or targets (miRNA inhibitors) in cancer and other diseases (Link et al., 2010; Kalla et al., 2015; Rupaimoole and Slack, 2017; Tili et al., 2017; Hossian et al., 2019; Chen et al., 2020).

An early encouraging work demonstrated that miRNAs could be isolated from circulating body fluids (Weber et al., 2010; Lu and Rothenberg, 2018; Lee, 2019). They exist and transfer 
stably outside the cell and in feces through vesicles. Exosomes (30-100 nm nanosized vesicles) derived from multiple cell types are capable of encapsulating protein and RNA molecules (Turchinovich et al., 2011; Celluzzi and Masotti, 2016; Yu et al., 2016; Bartel, 2018; Moloney et al., 2019). miRNA molecules have been observed to complete the intercellular communication in this way while preventing endogenous degradation (Turchinovich et al., 2011; Celluzzi and Masotti, 2016; Yu et al., 2016; Bartel, 2018; Moloney et al., 2019). miRNAs dysregulation observed in tissues and serum associated with disease activity was considered as a potential biomarker for clinical diagnosis (Link et al., 2010; Kalla et al., 2015; Rupaimoole and Slack, 2017; Tili et al., 2017; Hossian et al., 2019; Chen et al., 2020), but several issues have impeded the application of circulating miRNAs in clinical diagnosis (Wang et al., 2018; Saliminejad et al., 2019). In the host's gastrointestinal tract, fecal miRNA can be influenced by the alteration of gut microbial composition, which provides a new perspective to identify intestinal and microbiota healthiness (Carter et al., 2017; Horne et al., 2019; Tarallo et al., 2019; Sarshar et al., 2020).

\section{THE IMPLICATIONS OF MIRNA FOR THE HOST}

MicroRNAs play an important role in the host's gastrointestinal function. Numerous studies have revealed that miRNAs can participate in many aspects of intestinal functions, including proliferation and differentiation of cells, architecture, and barrier function of the host intestines (Zou et al., 2018; Ding et al., 2019). In 2010, McKenna et al. (2010) determined the complete miRNA transcriptome of the mouse intestinal epithelium cells. They found that miR-192 and let-7 presented high expression levels and observed reduction in goblet cells, increased apoptosis, and intestinal inflammation in the mice deficient of all miRNAs in the intestine. Part of the reason for the proliferation and differentiation of IECs is the precise regulation of key transcription factors, such as SOX9 (Peck et al., 2016). miR-30 could indirectly regulate SOX9 protein and thus mediate IEC homeostasis (Suez et al., 2019). Recent study showed that miR-381$3 \mathrm{p}$ directly suppressed nuclear receptor-related protein 1 (nurr1) translation (Liu et al., 2018). By inhibiting miR381-3p, nurr1-mediated IEC proliferation and barrier function were enhanced.

With the increasing recognition of the importance of miRNAs in the host's gastrointestinal tract function, the association between miRNAs and a wide variety of intestinal diseases have received more and more attention (Tables 1, 2). For example, colorectal cancer (CRC) is one of the most common malignant tumors. Multiple signaling pathways (WNT, EGFR, TGF- $\beta$, etc.) in CRC are regulated by miRNAs (Balacescu et al., 2018). They have been identified as oncomiRs and tumor suppressor miRNAs (Yamamoto and Mori, 2016; Balacescu et al., 2018; Shirafkan et al., 2018). Their deregulation contributes to the development, progression, and metastasis of CRC (Yamamoto and Mori, 2016; Balacescu et al., 2018; Shirafkan et al., 2018; To et al., 2018). Natural endogenous and stability make numerous of them therapeutic targets and potential biomarkers for CRC (Yamamoto and Mori, 2016; Shirafkan et al., 2018; To et al., 2018). The exploitation of favorable miRNAs delivery systems is likely to expand the options for CRC treatment. Similarly, many miRNAs could interfere in the pathogenesis of IBDs by regulating multiple pathways (Cao et al., 2017; Schönauen et al., 2018; Boros and Nagy, 2019; Feng et al., 2019). They were observed to play important roles in inflammation, fibrosis, autophagy, and cellular proliferation (Yamamoto and Mori, 2016; Balacescu et al., 2018; Shirafkan et al., 2018; To et al., 2018; Feng et al., 2019). According to Tian et al. (2019), miRNA 31 (miR31) expression was altered in inflamed mucosa of IBD patients via the NF-kB and STAT3 signaling pathways. This miRNA has the function of relieving inflammation and promoting epithelial cell regeneration. A microsphere delivery system has been developed based on this characteristic of miR31, which provided a new proposal for the treatment of IBD.

Notably, microbiota-derived extracellular vesicles (EVs) also play a major role in intercellular communication and signal transduction between gut microbiota and host cells. EVs included outer membrane vesicles (OMVs) and membrane vesicles (MVs) that are, respectively, liberated by Gram-negative and Grampositive bacteria. Various RNA species (e.g., mRNAs, miRNAs, tRNAs) are biologically active components of EVs, which may impact gene expression when being delivered to host cells (Ahmadi Badi et al., 2017; Felli et al., 2017; Macia et al., 2019; Sarshar et al., 2020). In a fascinating study, Fábrega et al. confirmed the role of OMVs in signal transduction between gut microbiota and host. OMVs produced by the probiotic Escherichia coli strain Nissle (EcN) 1917 induced the expression and secretion of several cytokines and chemokines in the ex vivo model (Fábrega et al., 2016). Overall, intestinal bacteria utilized OMVs as a significant strategy to communicate with the host and influence host responses.

\section{THE CORRELATION BETWEEN HOST MIRNA EXPRESSION LEVELS AND GUT MICROBIOTA PROFILES}

In addition to participating in the regulation of intestinal function, the close relationship between miRNAs and gut microbiota has also been confirmed (Masotti, 2012; Belcheva, 2017; Hoban et al., 2017; Yuan et al., 2018). A potential mechanism by which gut microbiota affect host physiology is to modulate host gene expression through miRNAs. Dalmasso et al. (2011) demonstrated that nine miRNAs were differently expressed in the colonized mice relative to germ-free mice. They confirmed that the potential targets of miRNAs overlapped with the dysregulated host genes during microbial colonization. Commensal bacteria might affect more host genes at the post-transcriptional level than expected. Singh et al. (2012) investigated the influence of endogenous microbiota on the overall expression of cecal miRNA in vivo by using germ-free and conventional mice. The result supported that cecal miRNA expression levels were modified by endogenous microbiota. The 
TABLE 1 | Circulating miRNAs and their possible/identified targets in different gastrointestinal disorders.

\begin{tabular}{|c|c|c|c|c|c|}
\hline $\begin{array}{l}\text { Circulating } \\
\text { miRNAs }\end{array}$ & Diseases & Up/down & $\begin{array}{l}\text { Potential/identified } \\
\text { target genes }\end{array}$ & Roles & References \\
\hline miR-25-3p & $\mathrm{CRC}$ & Up & KLF2, KLF4 & Promoting CRC vascular permeability and angiogenesis & Zeng et al., 2018 \\
\hline $\operatorname{miR}-106 b-3 p$ & CRC & Up & DLC-1 & Promoting $\mathrm{CRC}$ metastasis & Liu et al., 2020 \\
\hline miR-1229 & CRC & Up & HIPK2 & Promoting CRC angiogenesis & Hu et al., 2019 \\
\hline $\operatorname{miR}-590-5 p$ & CRC & Down & NF90 & Inhibiting CRC angiogenesis and metastasis & Zhou Q. et al., 2016 \\
\hline miR-622 & $\mathrm{CRC}$ & Down & CXCR4, K-Ras & Inhibiting $\mathrm{CRC}$ angiogenesis and metastasis & Fang et al., 2016, 2019 \\
\hline miR-382 & $\mathrm{CRC}$ & Down & NR2F2 & Inhibiting CRC cell growth and invasion & Zhou B. et al., 2016 \\
\hline miR-409-3p & $\mathrm{CRC}$ & Down & Beclin-1 & Inhibiting autophagy & Tan et al., 2016 \\
\hline miR-223 & $\mathrm{IBD}$ & Up & CLDN8 & Dysfunction of the intestinal epithelial barrier & Wang et al., 2016 \\
\hline miR-21 & $\mathrm{IBD}$ & Up & RhoB & Dysfunction of the intestinal epithelial barrier & Yang et al., 2013 \\
\hline miR-320 & $\mathrm{IBD}$ & Down & NOD2 & Disruption of immune homeostasis & Pierdomenico et al., 2016 \\
\hline $\operatorname{miR}-31$ & IBD & Up & IL6st, IL7r, IL17ra & Suppressing inflammation & Tian et al., 2019 \\
\hline
\end{tabular}

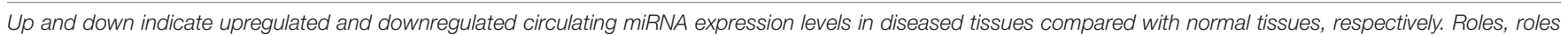
of miRNAs in the pathogenesis of gastrointestinal disorders; CRC, colorectal cancer; IBD, inflammatory bowel disease.

TABLE 2 | Fecal miRNAs and bacteria correlated with them in different gastrointestinal disorders.

\begin{tabular}{|c|c|c|c|c|}
\hline Fecal miRNAs & Diseases & Up/down & Correlated microbiome taxa & References \\
\hline miRNA-135b & CRC & Up & - & Wu et al., 2014 \\
\hline miR-21 & $\mathrm{CRC}$ & Up & - & Link et al., 2010 \\
\hline miR-106a & $\mathrm{CRC}$ & Up & - & Link et al., 2010 \\
\hline miR-182 & $\mathrm{CRC}$ & Up & Blautia & Yuan et al., 2018 \\
\hline miR-139 & $\mathrm{CRC}$ & Down & Blautia & Yuan et al., 2018 \\
\hline miR-155 & $\mathrm{IBD}$ & Up & - & Schönauen et al., 2018 \\
\hline miR-223 & $\mathrm{IBD}$ & Up & - & Schönauen et al., 2018 \\
\hline miR-199a & $\mathrm{IBD}$ & Up & - & Ji et al., 2018 \\
\hline miR-223-3p & $\mathrm{IBD}$ & Up & - & Ji et al., 2018 \\
\hline miR-1226 & $\mathrm{IBD}$ & Down & - & Ji et al., 2018 \\
\hline miR-515-5p & $\mathrm{IBD}$ & Down & - & Ji et al., 2018 \\
\hline
\end{tabular}

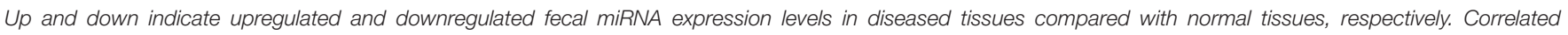
microbiome taxa, bacteria significantly correlated with fecal miRNAs; CRC, colorectal cancer; IBD, inflammatory bowel disease.

authors also discovered the proteins encoded by 34 putative miRNA targets involved in controlling intestinal barrier and immune regulation. Also, Xue et al. (2011) reported a study focusing on the roles of commensal bacteria in the regulation of intestinal gene expression. They found that commensal bacteria downregulated dendritic cell miR-10a expression via TLR-TLR ligand interactions through a MyD88-dependent pathway. IL$12 / \mathrm{IL}-23 \mathrm{p} 40$, a key molecule for innate immune responses to commensal bacteria, was identified as a target gene for miR10a. Compared with the control mice, colitis mice have higher levels of IL-12/IL-23p40 and lower levels of intestinal miR10a. Surprisingly, gut microbiota are also reported to influence host miRNA expression in other tissues and organs (Zhou G. et al., 2017; Allegra et al., 2020). Both the two studies have identified the effects of gut microbiota on the miRNA expression in the hippocampus and white adipocytes in mice, respectively (Chen J.J. et al., 2017; Virtue et al., 2019). An additional researcher claimed that after viral infection, the expression of miRNAs in the lungs of antibiotic-treated mice was altered, resulting in a reduction in host antiviral immunity (Pang et al., 2018). Accordingly, the aforementioned findings indicated that
miRNA could impact the host through participating in signal transmission, maintaining intestinal homeostasis.

\section{INTERACTIONS BETWEEN HOST mIRNAS AND BACTERIAL PATHOGENS}

miRNAs have also been recognized for their important role in the interactions between host and bacterial pathogens, either as an indispensable part of the host response to fight infection or as a molecular strategy utilized by bacterial pathogens to cause the dysregulation of host miRNA expression for their own benefit (Aguilar et al., 2019a,b). For example, miR-301b is involved in the augmentation of pro-inflammatory response during infection by Pseudomonas aeruginosa (Li X. et al., 2016). A target of miR-301b is c-Myb that can increase the expression of antiinflammatory cytokines IL- 4 and TGF- $\beta 1$. miR-301b suppresses anti-inflammatory response by targeting $\mathrm{c}-\mathrm{Myb}$ in response to $P$. aeruginosa infection. Wang et al. (2017) observed that miR143-3p expression was significantly upregulated in Helicobacter pylori-positive gastric cancer tissues. miR-143-3p targets AKT2, 
which induced cell apoptosis and negatively regulated tumor growth, migration, and invasion. Bacterial pathogens have evolved the ability to regulate host miRNAs to resist autophagy, thereby promoting the survival and reproduction of bacteria. miR-23a-5p was shown to facilitate bacterial survival and inhibit Mycobacterium tuberculosis-induced autophagy in macrophages by impacting the TLR2/MyD88/NF- $\kappa \mathrm{B}$ pathway ( $\mathrm{Gu}$ et al., 2017). miRNAs hav emerged as important participants in the interactions between host and bacterial pathogens, which suggests an underlying mechanism that miRNAs are transmitted between host and bacteria.

\section{FECAL MIRNA AND PLANT-DERIVED MIRNA AS USEFUL TOOLS FOR MAINTAINING GUT HOMEOSTASIS}

Actually, a bidirectional relationship exists between gut microbiome and miRNAs. An analysis of miRNA sequencing in human CRC tumor and normal tissues identified 76 differentially expressed miRNAs that are correlated with the abundance of microbes in the tumor microenvironment, including Firmicutes, Bacteroidetes, and Proteobacteria (Yuan et al., 2018). Moloney et al. (2018) proved that the expression of murine miRNAs produced by IECs in feces was influenced by the gut microbiota ( $\mathrm{Gu}$ et al., 2017). They discovered that the relative abundance of the phyla Bacteroidetes and Firmicutes was significantly correlated with the level of miR-141-3p and phyla Actinobacteria, Bacteroidetes, Cyanobacteria, Firmicutes, and Proteobacteria were significantly correlated with the level of miR-200a-3p. As a valuable tool, host miRNAs play significant roles in maintaining intestinal homeostasis (Link et al., 2010; Moloney et al., 2018; Yuan et al., 2018). Recent studies have illustrated the mechanism by which the host could foster favorable microbiota via fecal miRNA (Liu et al., 2016; Jiayi et al., 2019). An important work from Liu et al. introduced that fecal miRNAs are mainly derived from IECs and Hopx+ cells (Liu et al., 2016). miRNAs present in the feces have the ability to enter bacterial cells and specifically regulate gene transcripts, thereby affecting the growth of bacteria. miRNA mimics synthesized for oral administration could act on gut bacteria. Moreover, they found that IEC miRNA-deficient mice suffered from dysbiosis and that the fecal miRNA transplantation from wild-type mice restored the intestinal bacterial community. Recently, Liu and Weiner (2016) also reported that fecal miR-30d upregulated the expression of a lactase in Akkermansia muciniphila and increased this bacterial abundance in the gut, which suppressed multiple sclerosis in mouse model through the expansion of regulatory $\mathrm{T}$ cells (Liu and Weiner, 2016).

Dietary intervention is widely known as an important means for the host to maintain intestinal homeostasis (Liu et al., 2019). Another source of miRNAs that the host can utilize is plant-derived miRNAs. These miRNAs from food can also enter bacteria and regulate gene expression. Teng et al. revealed that miRNAs encapsulated in plant-derived exosome-like nanoparticles (ELNs) were taken up by gut bacteria, subsequently bound to bacterial mRNAs, and modified the composition of gut microbiota (Nagai et al., 2016). The priority for specific gut bacteria to absorb plant-derived ELNs depended on the lipid type of the outer membrane of ELNs. More importantly, miRNAs within ginger ELNs could enter Lactobacillus rhamnosus GG (LGG) and induce the IL-22 expression via the activation of the AHR pathway to enhance LGG-mediated inhibition of mouse colitis (Spinler et al., 2019). This result indicated that probiotics may be manipulated by miRNA originating from the host or diet.

In summary, these data demonstrated that the fecal miRNA and plant-derived miRNA contribute to the modification of the gut microbiome.

\section{EXPLORATION OF MIRNA-BASED MOLECULAR MECHANISM OF PROBIOTICS ACTION}

Host-microbiota interactions play a vital role in intestinal homeostasis, and miRNAs have been considered to be key molecular regulators for mediating such interactions (Behrouzi et al., 2020). The addition of probiotics can also interfere with these interactions and influence the expression of miRNAs (Teng et al., 2018). For instance, miRNAs involved in the alleviation of cecal inflammation are induced by probiotic Lactobacillus plantarum Z01 (Rodríguez-Nogales et al., 2018a). In IBD, probiotic EcN 1917 was reported to have the ability to regulate the miRNA expression levels that participated in the inflammatory response in colitic mice (Chen Q. et al., 2017). Similarly, Bacillus coagulans R11 may alter the structure of the bacterial community by influencing the host fecal miRNAs (Rodríguez-Nogales et al., 2018b). The ability to regulate miRNA expression by probiotics is of great significance for maintaining the intestinal microenvironment homeostasis. Notably, there is a highly complex relationship between miRNAs and intestinal diseases. However, few reports are available about the roles of miRNAs in shaping the gut microbiota as a therapy of diseases. Moreover, the capacity of the host to regulate gut microbiota in a miRNA-dependent manner remains poorly understood and may even be restricted. Considering that a health benefit was delivered to the host via the ingestion of probiotics, the effects of miRNAs may be strengthened by these beneficial microorganisms.

As mentioned above, miRNAs have emerged as important mediators of interactions between host and gut microbiota. Furthermore, probiotics have the ability to modulate miRNA expression levels, and we speculated that the potential mechanism of probiotics acting on the host may be associated with the modification of miRNAs (Figures 1, 3). Probiotics drive intestinal cells to produce miRNAs with important regulatory functions (such as intestinal anti-inflammatory effects) to act on host cells. On the other hand, probiotics impact the releases of fecal miRNAs from IECs, thereby prompting fecal miRNAs to penetrate into specific bacteria and regulate gene expression, with consequences for restoring the "signature" of gut microbiota. 


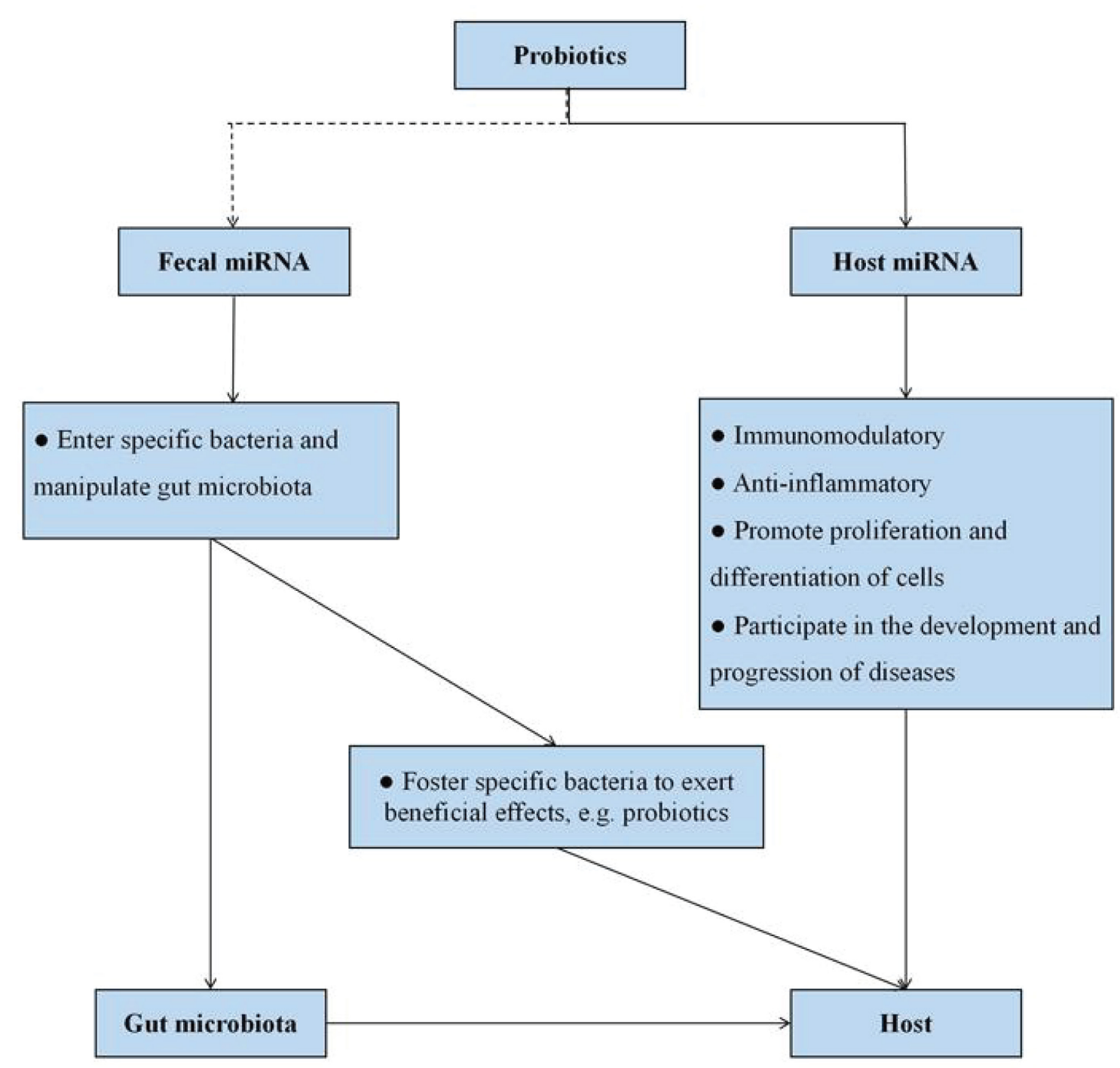

FIGURE 3 | Potential miRNA-based molecular mechanism of probiotics action. Probiotics can influence host miRNA expression, thereby favoring numerous host functions. On the other hand, host cells foster specific bacteria to control intestinal homeostasis and exert beneficial effects on the host itself via fecal miRNA and plant-derived miRNA. The dotted line represents the lack of conclusive evidence in the relationship at present.

Additionally, side effects of probiotics should not be ignored. Although probiotics are widely used and have considerable potential as preventive or therapeutic options against various gastrointestinal disorders, there are still a few reports indicating the potentially negative effects of probiotics. In fact, the reason for these conflicting clinical results is that the health benefits provided by probiotics to the host depend on many factors, including the strains, individuals, types of administration, or location within the intestines. At present, there is no sufficient and convincing evidence to demonstrate the mechanism of probiotics resulting in negative consequences in the host. The exact mechanisms of both positive and negative effects of probiotics should be further investigated and perfected. Here, this review aimed to open up a new direction for studying the molecular pathways involved in the probiotic mode of action. More explorations and evidence for the research about elucidating the probiotic mechanisms of action are desirable.

\section{FUTURE PERSPECTIVES}

In recent years, significant breakthroughs have been made in miRNA-based research field, especially in regard to the applications that provided opportunities for the treatment of numerous gastrointestinal diseases including CRC, IBD, etc. (Soroosh et al., 2018; Konno et al., 2019; Tian et al., 2019; Xing et al., 2019). Notably, a single miRNA can target multiple mRNAs, and one mRNA can become the target of numerous miRNAs. A wide range of miRNAs from mammalian cells constitute a complex regulatory network. In this context, it is particularly 
important to clarify the real targets with therapeutic effects. They will be valuable tools for the treatment and intervention of patients with various diseases. miRNA-based therapeutics are able to deliver miRNA mimics or anti-miRs to target tissues and target cells through advanced technologies. Several pathways have shown promise to enable this strategy to become a clinical reality. Simultaneously, the administration of fecal miRNA mimics or anti-miRs in vivo makes manipulation of the gut microbiota feasible. However, this concept is still relatively novel in the field, and the investigation of manipulating bacterial communities has just begun. Growing valuable insights will promote the cross-kingdom communication between host and gut microorganism to become a subject of intense exploration.

To date, accumulating evidence supported that miRNAs play central roles in mediating host-microbe interactions and can be used as biomarkers of intestinal and microbiota healthiness. Considering that probiotics are likely to be the regulators or amplifiers of miRNA expression levels, we believe that miRNA may be an important part of the molecular mechanism underlying how probiotics maintain gut homeostasis. In this point, the probiotics-miRNA-host/gut microbiota axis can serve as a new direction for the next phase of research but

\section{REFERENCES}

Abid, M. B., and Koh, C. J. (2019). Probiotics in health and disease: fooling Mother Nature? Infection 47, 911-917. doi: 10.1007/s15010-019-01351-0

Adak, A., and Khan, M. R. (2019). An insight into gut microbiota and its functionalities. Cell. Mol. Life Sci. 76, 473-493. doi: 10.1007/s00018-018-2943-4

Aguilar, C., Mano, M., and Eulalio, A. (2019a). MicroRNAs at the host-bacteria interface: host defense or bacterial offense. Trends Microbiol. 27, 206-218. doi: 10.1016/j.tim.2018.10.011

Aguilar, C., Mano, M., and Eulalio, A. (2019b). Multifaceted roles of microRNAs in host-bacterial pathogen interaction. Microbiol. Spectr. 7:31152522.

Agus, A., Planchais, J., and Sokol, H. (2018). Gut microbiota regulation of tryptophan metabolism in health and disease. Cell Host. Microbe. 23, 716-724. doi: 10.1016/j.chom.2018.05.003

Ahmadi Badi, S., Moshiri, A., Fateh, A., Rahimi Jamnani, F., Sarshar, M., Vaziri, F., et al. (2017). Microbiota-derived extracellular vesicles as new systemic regulators. Front. Microbiol. 8:1610. doi: 10.3389/fmicb.2017. 01610

Allaire, J. M., Crowley, S. M., Law, H. T., Chang, S. Y., Ko, H. J., Vallance, B. A., et al. (2018). The intestinal epithelium: central coordinator of mucosal immunity. Trends Immunol. 39, 677-696. doi: 10.1016/j.it.2018.04.002

Allegra, A., Musolino, C., Tonacci, A., Pioggia, G., Gangemi, S., et al. (2020). Interactions between the microRNAs and microbiota in cancer development: roles and therapeutic opportunities. Cancers 12:805. doi: 10 . 3390/cancers 12040805

Balacescu, O., Sur, D., Cainap, C., Visan, S., Cruceriu, D., Manzat-Saplacan, R., et al. (2018). The impact of miRNA in colorectal cancer progression and its liver metastases. Int. J. Mol. Sci. 19:3711. doi: 10.3390/ijms19123711

Balakrishnan, M., and Floch, M. H. (2012). Prebiotics, probiotics and digestive health. Curr. Opin. Clin. Nutr. Metab. Care 5, 580-585.

Bartel, D. P. (2018). Metazoan microRNAs. Cell 173, 20-51. doi: 10.1016/j.cell. 2018.03.006

Behrouzi, A., Ashrafian, F., Mazaheri, H., Lari, A., Nouri, M., Riazi Rad, F., et al. (2020). The importance of interaction between microRNAs and gut microbiota in several pathways. Microb. Pathog. 144:104200. doi: 10.1016/j.micpath.2020. 104200

Belcheva, A. (2017). MicroRNAs at the epicenter of intestinal homeostasis. Bioessays 39:28155997. with the caveat that this molecular mechanism of probiotics remains to be fully understood. Further work is needed to delineate the precise mechanism of how probiotics control miRNA expression levels and to obtain more knowledge about subsequently how these prominently expressed miRNAs mediate host-microbe interactions.

\section{AUTHOR CONTRIBUTIONS}

YZh, YZe, and XN contributed to the topic conception. YZh wrote the main manuscript text. YZe designed the figures. YZh and $M Z$ revised the manuscript. All authors read and approved the final manuscript.

\section{FUNDING}

The present study was supported by the National Natural Science Foundation of China (31970503), Sichuan Science and Technology Program (2021GJHZ0140 and 2019YFH0060), and Basic scientific research projects of Sichuan scientific research institutes (SASA201906).

Bermudez-Brito, M., Plaza-Díaz, J., Muñoz-Quezada, S., Gómez-Llorente, C., Gil, A., et al. (2019). Probiotic mechanisms of action. Early Hum. Dev. 135, 58-65. doi: 10.1016/j.earlhumdev.2019.05.010

Boros, É, and Nagy, I. (2019). The role of microRNAs upon epithelial-tomesenchymal transition in inflammatory bowel disease. Cells 8:1461. doi: 10 . 3390/cells8111461

Brown, E. M., Manish, S., and Brett, F. (2013). The role of the immune system in governing host-microbe interactions in the intestine. Nat. Immunol. 14, 660-667. doi: 10.1038/ni.2611

Cao, B., Zhou, X., Ma, J., Zhou, W., Yang, W., Fan, D., et al. (2017). Role of miRNAs in inflammatory bowel disease. Dig. Dis. Sci. 62, 14261438.

Carter, J. V., Galbraith, N. J., Yang, D., Burton, J. F., Walker, S. P., Galandiuk, S., et al. (2017). Blood-based microRNAs as biomarkers for the diagnosis of colorectal cancer: a systematic review and meta-analysis. Br. J. Cancer 116, 762-774. doi: 10.1038/bjc.2017.12

Celiker, C., and Kalkan, R. (2020). Genetic and epigenetic perspective of microbiota. Appl. Microbiol. Biotechnol. 104, 8221-8229. doi: 10.1007/s00253020-10849-9

Celluzzi, A., and Masotti, A. (2016). How our other genome controls our epi-genome. Trends Microbiol. 24, 777-787. doi: 10.1016/j.tim.2016. 05.005

Chang, C. S., and Kao, C. Y. (2019). Current understanding of the gut microbiota shaping mechanisms. J. Biomed. Sci. 26:59.

Chen, J. J., Zeng, B. H., Li, W. W., Zhou, C. J., Fan, S. H., Cheng, K., et al. (2017). Effects of gut microbiota on the microRNA and mRNA expression in the hippocampus of mice. Behav. Brain Res. 322, 34-41. doi: 10.1016/j.bbr.2017. 01.021

Chen, P., Zhou, G., Lin, J., Li, L., Zeng, Z., Chen, M., et al. (2020). Serum biomarkers for inflammatory bowel disease. Front. Med. 7:123. doi: doi: 10.3389/fmed.2020.00123

Chen, Q., Tong, C., Ma, S., Zhou, L., Zhao, L., Zhao, X., et al. (2017). Involvement of microRNAs in probiotics-induced reduction of the cecal inflammation by Salmonella Typhimurium. Front. Immunol. 8:704. doi: 10.3389/fimmu.2017. 00704

Chu, H., and Mazmanian, S. K. (2013). Innate immune recognition of the microbiota promotes host-microbial symbiosis. Nat. Immunol. 14, 668-675. doi: $10.1038 /$ ni.2635 
Coyte, K. Z., Schluter, J., and Foster, K. R. (2015). The ecology of the microbiome: networks, competition, and stability. Science 350, 663-666. doi: 10.1126/ science.aad 2602

Cremon, C., Barbaro, M. R., Ventura, M., and Barbara, G. (2018). Pre- and probiotic overview. Curr. Opin. Pharmacol. 43, 87-92. doi: 10.1016/j.coph. 2018.08.010

Cristofori, F., Indrio, F., Miniello, V. L., De Angelis, M., and Francavilla, R. (2018). Probiotics in celiac disease. Nutrients 10:1824. doi: 10.3390/nu10121824

Cuevas-Sierra, A., Ramos-Lopez, O., Riezu-Boj, J. I., Milagro, F. I., and Martinez, J. A. (2019). Diet, gut microbiota, and obesity: links with host genetics and epigenetics and potential applications. Adv. Nutr. 10, S17-S30.

Dalmasso, G., Nguyen, H. T., Yan, Y., Laroui, H., Charania, M. A., Ayyadurai, S., et al. (2011). Microbiota modulate host gene expression via microRNAs. PLoS One. 6:e19293. doi: 10.1371/journal.pone.0019293

Ding, S., Liu, G., Jiang, H., and Fang, J. (2019). MicroRNA determines the fate of intestinal epithelial cell differentiation and regulates intestinal diseases. Curr. Protein. Pept. Sci. 20, 666-673. doi: 10.2174/1389203720666190125110626

Dobson, A., Cotter, P. D., Ross, R. P., and Hill, C. (2012). Bacteriocin production: a probiotic trait? Appl. Environ. Microbiol. 78, 1-6. doi: 10.1128/aem.05576-11

Du, L., Liu, F., Ju, X., and Huo, G. (2010). Adhesion capability of first two domains at $\mathrm{N}$ terminus of NP_785232 protein and their interaction with a UVabsorbing component from human mucus. Lett. Appl. Microbiol. 51, 400-405. doi: 10.1111/j.1472-765x.2010.02911.x

El Kaoutari, A., Armougom, F., Gordon, J. I., Raoult, D., and Henrissat, B. (2013). The abundance and variety of carbohydrate-active enzymes in the human gut microbiota. Nat. Rev. Microbiol. 11, 497-504. doi: 10.1038/nrmicro3050

Fábrega, M. J., Aguilera, L., Giménez, R., Varela, E., Alexandra Cañas, M., Antolín, M., et al. (2016). Activation of immune and defense responses in the intestinal mucosa by outer membrane vesicles of commensal and probiotic Escherichia coli Strains. Front. Microbiol. 7:705. doi: 10.3389/fmicb.2016.00705

Fang, Y., Sun, B., Li, Z., Chen, Z., and Xiang, J. (2016). MiR-622 inhibited colorectal cancer occurrence and metastasis by suppressing K-Ras. Mol. Carcinog. 55, 1369-1377. doi: 10.1002/mc.22380

Fang, Y., Sun, B., Wang, J., and Wang, Y. (2019). miR-622 inhibits angiogenesis by suppressing the CXCR4-VEGFA axis in colorectal cancer. Gene 699, 37-42. doi: 10.1016/j.gene.2019.03.004

Felli, C., Baldassarre, A., and Masotti, A. (2017). Intestinal and circulating microRNAs in coeliac disease. Int. J. Mol. Sci. 18:1907. doi: 10.3390/ ijms 18091907

Feng, Y., Zhang, Y., Zhou, D., Chen, G., and Li, N. (2019). MicroRNAs, intestinal inflammatory and tumor. Bioorg. Med. Chem. Lett. 29, 2051-2058. doi: 10.1016/ j.bmcl.2019.06.013

Fischer, S. E. J. (2015). RNA interference and microRNA-mediated silencing. Curr. Protoc. Mol. Biol. 112, 26.1.1-26.1.5.

Forgie, A. J., Fouhse, J. M., and Willing, B. P. (2019). Diet-microbe-host interactions that affect gut mucosal integrity and infection resistance. Front. Immunol. 10:1802. doi: 10.3389/fimmu.2019.01802

Gentile, C. L., and Weir, T. L. (2018). The gut microbiota at the intersection of diet and human health. Science 362, 776-780. doi: 10.1126/science.aau5812

Gómez-Llorente, C., Muñoz, S., and Gil, A. (2010). Role of Toll-like receptors in the development of immunotolerance mediated by probiotics. Proc. Nutr. Soc. 69, 381-389. doi: 10.1017/s0029665110001527

Gresse, R., Chaucheyras-Durand, F., Fleury, M. A., Van de Wiele, T., Forano, E., Blanquet-Diot, S., et al. (2017). Gut microbiota dysbiosis in postweaning piglets: understanding the keys to health. Trends Microbiol. 25, 851-873. doi: 10.1016/j.tim.2017.05.004

Gu, X., Gao, Y., Mu, D. G., and Fu, E. Q. (2017). MiR-23a-5p modulates mycobacterial survival and autophagy during mycobacterium tuberculosis infection through TLR2/MyD88/NF-кB pathway by targeting TLR2. Exp. Cell Res. 354, 71-77. doi: 10.1016/j.yexcr.2017.03.039

Gura, T. (2014). Nature's first functional food. Science 345, 747-749. doi: 10.1126/ science.345.6198.747

Hasan, N., and Yang, H. (2019). Factors affecting the composition of the gut microbiota, and its modulation. PeerJ. 7:e7502. doi: 10.7717/peerj.7502

Hill, C., Guarner, F., Reid, G., Gibson, G. R., Merenstein, D. J., Pot, B., et al. (2014). Expert consensus document. Te International Scientifc Association for Probiotics and Prebiotics consensus statement on the scope and appropriate use of the term probiotic. Nat. Rev. Gastroenterol. Hepatol. 11, 506-514. doi: 10.1038/nrgastro.2014.66

Hoban, A. E., Stilling, R. M., Moloney, G. M., Moloney, R. D., and Shanahan, F. (2017). Microbial regulation of microRNA expression in the amygdala and prefrontal cortex. Microbiome 5:102.

Horne, R., St Pierre, J., Odeh, S., Surette, M., and Foster, J. A. (2019). Microbe and host interaction in gastrointestinal homeostasis. Psychopharmacology 236, 1623-1640. doi: 10.1007/s00213-019-05218-y

Hossian, A. K. M. N., Mackenzie, G. G., and Mattheolabakis, G. (2019). miRNAs in gastrointestinal diseases: can we effectively deliver RNA-based therapeutics orally? Nanomedicine 14, 2873-2889. doi: 10.2217/nnm-2019-0180

Hu, H. Y., Chen-Huan, Y., Huan-Huan, Z., Song-Zhao, Z., and Wen-Ying, Y. (2019). Exosomal miR-1229 derived from colorectal cancer cells promotes angiogenesis by targeting HIPK2. Int. J. Biol. Macromol. 132, 470-477. doi: 10.1016/j.ijbiomac.2019.03.221

Ji, Y., Li, X., Zhu, Y., Li, N., Zhang, N., Niu, M., et al. (2018). Faecal microRNA as a biomarker of the activity and prognosis of inflammatory bowel diseases. Biochem. Biophys. Res. Commun. 503, 2443-2450. doi: 10.1016/j.bbrc.2018.06. 174

Jiayi, D., Jesse, W. T., and Li-Fan, L. (2019). miRNA-microbiota interaction in gut homeostasis and colorectal cancer. Trends Cancer 5, 666-669. doi: 10.1016/j. trecan.2019.08.003

Kalla, R., Ventham, N. T., Kennedy, N. A., Quintana, J. F., Nimmo, E. R., Buck, A. H., et al. (2015). MicroRNAs: new players in IBD. Gut 64, 504-517. doi: 10.1136/gutjnl-2014-307891

Khoruts, A. (2018). Targeting the microbiome: from probiotics to fecal microbiota transplantation. Genome Med. 10:80.

Kiousi, D. E., Karapetsas, A., Karolidou, K., Panayiotidis, M. I., Pappa, A., Galanis, A., et al. (2019). Probiotics in extraintestinal diseases: current trends and new directions. Nutrients 11:788. doi: 10.3390/nu11040788

Konno, M., Koseki, J., Asai, A., Yamagata, A., Shimamura, T., Motooka, D., et al. (2019). Distinct methylation levels of mature microRNAs in gastrointestinal cancers. Nat. Commun. 10:3888.

Kothari, D., Patel, S., and Kim, S. K. (2019). Probiotic supplements might not be universally-effective and safe: A review. Biomed. Pharmacother. 111, 537-547. doi: 10.1016/j.biopha.2018.12.104

Langella, P., and Chatel, J. M. (2019). Risk assessment of probiotics use requires clinical parameters. Nat. Rev. Gastroenterol. Hepatol. 16, 202-204. doi: 10.1038/ s41575-019-0111-4

LeBlanc, J. G., Chain, F., Martín, R., Bermúdez-Humarán, L. G., Courau, S., Langella, P., et al. (2017). Beneficial effects on host energy metabolism of shortchain fatty acids and vitamins produced by commensal and probiotic bacteria. Microb. Cell Fact. 16:79.

Lee, E. S., Song, E. J., Nam, Y. D., and Lee, S. Y. (2018). Probiotics in human health and disease: from nutribiotics to pharmabiotics. J. Microbiol. 56, 773-782. doi: 10.1007/s12275-018-8293-y

Lee, H. (2019). Microbe-host communication by small RNAs in extracellular vesicles: vehicles for transkingdom RNA transportation. Int. J. Mol. Sci. 20:1487. doi: 10.3390/ijms20061487

Li, D., Wang, P., Wang, P., Hu, X., and Chen, F. (2016). The gut microbiota: A treasure for human health. Biotechnol. Adv. 34, 1210-1224. doi: 10.1016/j. biotechadv.2016.08.003

Li, X., He, S., Li, R., Zhou, X., Zhang, S., Yu, M., et al. (2016). Pseudomonas aeruginosa infection augments inflammation through miR-301b repression of c-Myb-mediated immune activation and infiltration. Nat. Microbiol. 1:16132.

Link, A., Balaguer, F., Shen, Y., Nagasaka, T., Lozano, J. J., Boland, C. R., et al. (2010). Fecal MicroRNAs as novel biomarkers for colon cancer screening. Cancer Epidemiol. Biomar. Prev. 19, 1766-1774. doi: 10.1158/1055-9965.epi10-0027

Liu, H., Liu, Y., Sun, P., Leng, K., Xu, Y., Mei, L., et al. (2020). Colorectal cancerderived exosomal miR-106b-3p promotes metastasis by down-regulating DLC1 expression. Clin. Sci. 134, 419-434. doi: 10.1042/cs20191087

Liu, L., Yao, J., Li, Z., Zu, G., Feng, D., Li, Y., et al. (2018). miR-381-3p knockdown improves intestinal epithelial proliferation and barrier function after intestinal ischemia/reperfusion injury by targeting nurr1. Cell Death Dis. 9:411.

Liu, S. (2016). The development of our organ of other kinds-the gut microbiota. Front. Microbiol. 7:2107. doi: 10.3389/fmicb.2016.02107 
Liu, S., and Weiner, H. L. (2016). Control of the gut microbiome by fecal microRNA. Microb. Cell. 3, 176-177. doi: 10.15698/mic2016.04.492

Liu, S., da Cunha, A. P., Rezende, R. M., Cialic, R., Wei, Z., Bry, L., et al. (2016). The host shapes the gut microbiota via fecal microRNA. Cell Host. Microbe. 19, 32-43. doi: 10.1016/j.chom.2015.12.005

Liu, S., Rezende, R. M., Moreira, T. G., Tankou, S. K., Cox, L. M., Wu, M., et al. (2019). Oral administration of miR-30d from feces of MS patients suppresses MS-like symptoms in mice by expanding Akkermansia muciniphila. Cell Host. Microbe. 26, 779-794.e.

Liwinski, T., and Elinav, E. (2020). Harnessing the microbiota for therapeutic purposes. Am. J. Transplant. 20, 1482-1488. doi: 10.1111/ajt.15753

Long, S. L., Gahan, C. G. M., and Joyce, S. A. (2017). Interactions between gut bacteria and bile in health and disease. Mol. Aspects Med. 56, 54-65. doi: 10.1016/j.mam.2017.06.002

Lozupone, C. A., Stombaugh, J. I., Gordon, J. I., Jansson, J. K., and Knight, R. (2012). Diversity, stability and resilience of the human gut microbiota. Nature 489, 220-230. doi: 10.1038/nature11550

Lu, T. X., and Rothenberg, M. E. (2018). MicroRNA. J. Allergy Clin. Immunol. 141, 1202-1207.

Macia, L., Nanan, R., Hosseini-Beheshti, E., Grau, G. E., et al. (2019). Hostand microbiota-derived extracellular vesicles, immune function, and disease development. Int. J. Mol. Sci. 21:107. doi: 10.3390/ijms21010107

Markowiak, P., and Śliżewska, K. (2017). Effects of probiotics, prebiotics, and synbiotics on human health. Nutrients 9:1021. doi: 10.3390/nu9091021

Maslowski, K. M. (2019). Metabolism at the centre of the host-microbe relationship. Clin. Exp. Immunol. 197, 193-204. doi: 10.1111/cei.13329

Masotti, A. (2012). Interplays between gut microbiota and gene expression regulation by miRNAs. Front. Cell Infect. Microbiol. 2:137. doi: 10.3389/fcimb. 2012.00137

Matsuki, T., Pédron, T., Regnault, B., Mulet, C., Hara, T., Sansonetti, P. J., et al. (2013). Epithelial cell proliferation arrest induced by lactate and acetate from Lactobacillus casei and Bifidobacterium breve. PLoS One. 8:e63053. doi: 10.1371/ journal.pone.0063053

McKenna, L. B., Schug, J., Vourekas, A., McKenna, J. B., Bramswig, N. C., Friedman, J. R., et al. (2010). MicroRNAs control intestinal epithelial differentiation, architecture, and barrier function. Gastroenterology 139, 16541664.e1.

Milani, C., Duranti, S., Bottacini, F., Casey, E., Turroni, F., Mahony, J., et al. (2017). The first microbial colonizers of the human gut: composition, activities, and health implications of the infant gut microbiota. Microbiol. Mol. Biol. Rev. 81, e36-e17.

Mohr, A. M., and Mott, J. L. (2015). Overview of microRNA biology. Semin. Liver Dis. 35, 3-11. doi: 10.1007/978-3-540-78709-9_1

Moloney, G. M., Dinan, T. G., Clarke, G., and Cryan, J. F. (2019). Microbial regulation of microRNA expression in the brain-gut axis. Curr. Opin. Pharmacol. 48, 120-126. doi: 10.1016/j.coph.2019.08.005

Moloney, G. M., Viola, M. F., Hoban, A. E., Dinan, T. G., Cryan, J. F., et al. (2018). Faecal microRNAs: indicators of imbalance at the host-microbe interface? Benef. Microbes. 9, 175-183. doi: 10.3920/bm2017.0013

Nagai, M., Obata, Y., Takahashi, D., and Hase, K. (2016). Fine-tuning of the mucosal barrier and metabolic systems using the diet-microbial metabolite axis. Int. Immunopharmacol. 37, 79-86. doi: 10.1016/j.intimp.2016.04.001

Pang, P., Yu, B., Shi, Y., Deng, L., Xu, H., Wu, S., et al. (2018). Alteration of intestinal flora stimulates pulmonary microRNAs to interfere with host antiviral immunity in influenza. Molecules 23:3151. doi: 10.3390/molecules23123151

Parasar, B., Zhou, H., Xiao, X., Shi, Q., Brito, I. L., and Chang, P. V. (2019). Chemoproteomic profiling of gut microbiota-associated bile salt hydrolase activity. ACS Cent Sci. 5, 867-873.

Parker, A., Lawson, M. A. E., Vaux, L., and Pin, C. (2018). Host-microbe interaction in the gastrointestinal tract. Environ. Microbiol. 20, 2337-2353. doi: 10.1111/ $1462-2920.13926$

Peck, B. C., Sincavage, J., Feinstein, S., Mah, A. T., Simmons, J. G., Lund, P. K., et al. (2016). miR-30 family controls proliferation and differentiation of intestinal epithelial cell models by directing a broad gene expression program that includes SOX9 and the ubiquitin ligase pathway. J. Biol. Chem. 291, 15975-15984. doi: 10.1074/jbc.m116.733733

Pierdomenico, M., Cesi, V., Cucchiara, S., Vitali, R., Prete, E., Costanzo, M., et al. (2016). NOD2 is regulated by miR-320 in physiological conditions but this control is altered in inflamed tissues of patients with inflammatory bowel disease. Inflamm. Bowel Dis. 22, 315-326. doi: 10.1097/mib.0000000000000659

Quigley, E. M. M., and Gajula, P. (2020). Recent advances in modulating the microbiome. F1000Res. 9:F1000FacultyRev-46.

Rodríguez-Nogales, A., Algieri, F., Garrido-Mesa, J., Vezza, T., Utrilla, M. P., Chueca, N., et al. (2018a). Intestinal anti-inflammatory effect of the probiotic Saccharomyces boulardii in DSS-induced colitis in mice: Impact on microRNAs expression and gut microbiota composition. J. Nutr. Biochem. 61, 129-139. doi: 10.1016/j.jnutbio.2018.08.005

Rodríguez-Nogales, A., Algieri, F., Garrido-Mesa, J., Vezza, T., Utrilla, M. P., Chueca, N., et al. (2018b). The administration of Escherichia coli Nissle 1917 ameliorates development of DSS-induced colitis in mice. Front. Pharmacol. 9:468. doi: 10.3389/fphar.2018.00468

Rooks, M. G., and Garrett, W. S. (2016). Gut microbiota, metabolites and host immunity. Nat. Rev. Immunol. 16, 341-352. doi: 10.1038/nri.2016.42

Rowland, I., Gibson, G., Heinken, A., Scott, K., Swann, J., Thiele, I., et al. (2018). Gut microbiota functions: metabolism of nutrients and other food components. Eur. J. Nutr. 57, 1-24. doi: 10.1007/s00394-017-1445-8

Rupaimoole, R., and Slack, F. J. (2017). MicroRNA therapeutics: towards a new era for the management of cancer and other diseases. Nat. Rev. Drug Discov. 16, 203-222. doi: 10.1038/nrd.2016.246

Saliminejad, K., Khorram Khorshid, H. R., and Ghaffari, S. H. (2019). Why have microRNA biomarkers not been translated from bench to clinic? Fut. Oncol. 15, 801-803. doi: $10.2217 /$ fon-2018-0812

Salvucci, E. (2019). The human-microbiome superorganism and its modulation to restore health. Int. J. Food Sci. Nutr. 70, 781-795. doi: 10.1080/09637486.2019. 1580682

Sanders, M. E., Merenstein, D. J., Reid, G., Gibson, G. R., and Rastall, R. A. (2019). Probiotics and prebiotics in intestinal health and disease: from biology to the clinic. Nat. Rev. Gastroenterol. Hepatol. 16, 605-616. doi: 10.1038/s41575-0190173-3

Sarshar, M., Scribano, D., Ambrosi, C., Palamara, A. T., and Masotti, A. (2020). Fecal microRNAs as innovative biomarkers of intestinal diseases and effective players in host-microbiome interactions. Cancers 12:E2174.

Schönauen, K., Le, N., von Arnim, U., Schulz, C., Malfertheiner, P., Link, A., et al. (2018). Circulating and fecal microRNAs as biomarkers for inflammatory bowel diseases. Inflamm. Bowel Dis. 24, 1547-1557. doi: 10.1093/ibd/izy046

Sehrawat, N., Yadav, M., Singh, M., Kumar, V., Sharma, V. R., Sharma, A. K., et al. (2020). Probiotics in microbiome ecological balance providing a therapeutic window against cancer. Semin. Cancer Biol. 20, S1044-S1579.

Shirafkan, N., Mansoori, B., Mohammadi, A., Shomali, N., Ghasbi, M., Baradaran, B., et al. (2018). MicroRNAs as novel biomarkers for colorectal cancer: new outlooks. Biomed. Pharmacother. 97, 1319-1330. doi: 10.1016/j.biopha.2017. 11.046

Singh, N., Shirdel, E. A., Waldron, L., Zhang, R. H., Jurisica, I., Comelli, E. M., et al. (2012). The murine caecal microRNA signature depends on the presence of the endogenous microbiota. Int. J. Biol. Sci. 8, 171-186. doi: 10.7150/ijbs.8.171

Soroosh, A., Koutsioumpa, M., Pothoulakis, C., Iliopoulos, D., et al. (2018). Functional role and therapeutic targeting of microRNAs in inflammatory bowel disease. Am. J. Physiol. Gastrointest Liver Physiol. 314, G256-G262.

Spinler, J. K., Karri, V., and Hirschi, K. D. (2019). Planting the microbiome. Trends Microbiol. 27, 90-93. doi: 10.1016/j.tim.2018.12.001

Suez, J., Zmora, N., Segal, E., and Elinav, E. (2019). The pros, cons, and many unknowns of probiotics. Nat. Med. 25, 716-729. doi: 10.1038/s41591-0190439-x

Tan, S., Shi, H., Ba, M., Lin, S., Tang, H., Zeng, X., et al. (2016). miR-409-3p sensitizes colon cancer cells to oxaliplatin by inhibiting Beclin-1-mediated autophagy. Int. J. Mol. Med. 37, 1030-1038. doi: 10.3892/ijmm.2016.2492

Tang, L. (2019). Diet influences microbe-host interaction. Nat. Methods. 16:361. doi: 10.1038/s41592-019-0413-z

Tarallo, S., Ferrero, G., Gallo, G., Francavilla, A., Clerico, G., Realis Luc, A., et al. (2019). Altered fecal small RNA profiles in colorectal cancer reflect gut microbiome composition in stool samples. mSystems 4, e289-e219.

Teng, Y., Ren, Y., Sayed, M., Hu, X., Lei, C., Kumar, A., et al. (2018). Plantderived exosomal microRNAs shape the gut microbiota. Cell Host. Microbe. 24, 637-652.e.

Tian, Y., Xu, J., Li, Y., Zhao, R., Du, S., Lv, C., et al. (2019). MicroRNA-31 reduces inflammatory signaling and promotes regeneration in colon epithelium, and 
delivery of mimics in microspheres reduces colitis in mice. Gastroenterology 156, 2281-2296.e.

Tili, E., Michaille, J. J., Piurowski, V., Rigot, B., Croce, C. M., et al. (2017). MicroRNAs in intestinal barrier function, inflammatory bowel disease and related cancers-their effects and therapeutic potentials. Curr. Opin. Pharmacol. 37, 142-150. doi: 10.1016/j.coph.2017.10.010

To, K. K., Tong, C. W., Wu, M., and Cho, W. C. (2018). MicroRNAs in the prognosis and therapy of colorectal cancer: From bench to bedside. World J. Gastroenterol. 24, 2949-2973. doi: 10.3748/wjg.v24.i27.2949

Turchinovich, A., Weiz, L., Langheinz, A., and Burwinkel, B. (2011). Characterization of extracellular circulating microRNA. Nucleic Acids Res. 39, 7223-7233. doi: 10.1093/nar/gkr254

Virtue, A. T., McCright, S. J., Wright, J. M., Jimenez, M. T., Mowel, W. K., Kotzin, J. J., et al. (2019). The gut microbiota regulates white adipose tissue inflammation and obesity via a family of microRNAs. Sci. Transl. Med. 11:eaav1892. doi: 10.1126/scitranslmed.aav1892

Wan, M. L. Y., Forsythe, S. J., and El-Nezami, H. (2019). Probiotics interaction with foodborne pathogens: a potential alternative to antibiotics and future challenges. Crit. Rev. Food Sci. Nutr. 59, 3320-3333. doi: 10.1080/10408398. 2018.1490885

Wang, F., Liu, J., Zou, Y., Jiao, Y., Huang, Y., Fan, L., et al. (2017). MicroRNA-143$3 p$, up-regulated in $H$. pylori-positive gastric cancer, suppresses tumor growth, migration and invasion by directly targeting AKT2. Oncotarget 8, 28711-28724. doi: 10.18632/oncotarget.15646

Wang, H., Chao, K., Ng, S. C., Bai, A. H., Yu, Q., Yu, J., et al. (2016). Proinflammatory miR-223 mediates the cross-talk between the IL23 pathway and the intestinal barrier in inflammatory bowel disease. Genome Biol. 17:58.

Wang, H., Peng, R., Wang, J., Qin, Z., and Xue, L. (2018). Circulating microRNAs as potential cancer biomarkers: the advantage and disadvantage. Clin. Epigenet. 10:59.

Weber, J. A., Baxter, D. H., Zhang, S., Huang, D. Y., Huang, K. H., Lee, M. J., et al. (2010). The microRNA spectrum in 12 body fluids. Clin. Chem. 56, 1733-1741. doi: 10.1373/clinchem.2010.147405

Wu, C. W., Ng, S. C., Dong, Y., Tian, L., Ng, S. S., Leung, W. W., et al. (2014). Identification of microRNA-135b in stool as a potential noninvasive biomarker for colorectal cancer and adenoma. Clin. Cancer Res. 20, 2994-3002. doi: 10.1158/1078-0432.ccr-13-1750

Xing, S. C., Xing, S. C., Huang, C. B., Mi, J. D., Wu, Y. B., and Liao, X. D. (2019). Bacillus coagulans $\mathrm{R} 11$ maintained intestinal villus health and decreased intestinal injury in lead-exposed mice by regulating the intestinal microbiota and influenced the function of faecal microRNAs. Environ. Pollut. 255:113139. doi: 10.1016/j.envpol.2019.113139

Xue, X., Feng, T., Yao, S., Wolf, K. J., Liu, C. G., Liu, X., et al. (2011). Microbiota downregulates dendritic cell expression of miR-10a, which targets IL-12/IL-23p40. J. Immunol. 187, 5879-5886. doi: 10.4049/jimmunol.11 00535

Yadav, M., and Pratyoosh, S. (2019). Recent systems biology approaches for probiotics use in health aspects: a review. 3 Biotech. 9:448.

Yamamoto, H., and Mori, M. (2016). MicroRNAs as therapeutic targets and colorectal cancer therapeutics. Adv. Exp. Med. Biol. 937, 239-247. doi: 10.1007/ 978-3-319-42059-2_13
Yan, F., and Polk, D. B. (2020). Probiotics and probiotic-derived functional factors-mechanistic insights into applications for intestinal homeostasis. Front. Immunol. 11:1428. doi: 10.3389/fimmu.2020.01428

Yang, Y., Ma, Y., Shi, C., Chen, H., Zhang, H., Chen, N., et al. (2013). Overexpression of miR-21 in patients with ulcerative colitis impairs intestinal epithelial barrier function through targeting the Rho GTPase RhoB. Biochem. Biophys. Res. Commun. 434, 746-752. doi: 10.1016/j.bbrc.2013.03.122

Yousefi, B., Eslami, M., Ghasemian, A., Kokhaei, P., Salek Farrokhi, A., and Darabi, N. (2019). Probiotics importance and their immunomodulatory properties. J. Cell Physiol. 234, 8008-8018. doi: 10.1002/jcp.27559

Yu, X., Odenthal, M., and Fries, J. W. (2016). Exosomes as miRNA carriers: formation-function-future. Int. J. Mol. Sci. 17:2028. doi: 10.3390/ijms17122028

Yuan, C., Burns, M. B., Subramanian, S., and Blekhman, R. (2018). Interaction between host microRNAs and the gut microbiota in colorectal cancer. $m$ Systems 3, e205-e217.

Zeng, Z., Li, Y., Pan, Y., Lan, X., Song, F., Sun, J., et al. (2018). Cancer-derived exosomal miR-25-3p promotes pre-metastatic niche formation by inducing vascular permeability and angiogenesis. Nat. Commun. 9:5395.

Zhang, C. X., Wang, H. Y., and Chen, T. X. (2019). Interactions between intestinal microflora/probiotics and the immune system. Biomed. Res. Int. 2019:67 64919.

Zhou, B., Song, J., Han, T., Huang, M., Jiang, H., Qiao, H., et al. (2016). MiR-382 inhibits cell growth and invasion by targeting NR2F2 in colorectal cancer. Mol. Carcinog. 55, 2260-2267. doi: 10.1002/mc.22466

Zhou, G., Zhou, Y., and Chen, X. (2017). New insight into inter-kingdom communication: horizontal transfer of mobile small RNAs. Front. Microbiol. 8:768. doi: $10.3389 /$ fmicb. 2017.00768

Zhou, M., He, J., Shen, Y., Zhang, C., Wang, J., Chen, Y., et al. (2017). New frontiers in genetics, gut microbiota, and immunity: a rosetta stone for the pathogenesis of inflammatory bowel disease. Biomed. Res. Int. 2017:8201672.

Zhou, Q., Zhu, Y., Wei, X., Zhou, J., Chang, L., Sui, H., et al. (2016). MiR-590$5 \mathrm{p}$ inhibits colorectal cancer angiogenesis and metastasis by regulating nuclear factor 90/vascular endothelial growth factor A axis. Cell Death Dis. 7:e2413. doi: $10.1038 /$ cddis. 2016.306

Zimmermann, M., Zimmermann-Kogadeeva, M., and Wegmann, R. (2019). Mapping human microbiome drug metabolism by gut bacteria and their genes. Nature 570, 462-467. doi: 10.1038/s41586-019-1291-3

Zou, L., Xiong, X., Wang, K., and Yin, Y. (2018). MicroRNAs in the intestine: role in renewal, homeostasis, and inflammation. Curr. Mol. Med. 18, 190-198. doi: $10.2174 / 1566524018666180907163638$

Conflict of Interest: The authors declare that the research was conducted in the absence of any commercial or financial relationships that could be construed as a potential conflict of interest.

Copyright ( 2021 Zhao, Zeng, Zeng, Wang, Zhou, Sun, Xin, Khalique, Rajput, Pan, Shu, Jing and Ni. This is an open-access article distributed under the terms of the Creative Commons Attribution License (CC BY). The use, distribution or reproduction in other forums is permitted, provided the original author(s) and the copyright owner(s) are credited and that the original publication in this journal is cited, in accordance with accepted academic practice. No use, distribution or reproduction is permitted which does not comply with these terms. 